\title{
Jean-Philippe Toussaint : la forme et la mélancolie
}

\author{
Roger-Michel Allemand \\ Laboratoire Babel - Université du Sud Toulon - Var
}

«Selon la doctrine idéaliste, les verbes vivre et rêver sont rigoureusement synonymes; de milliers d'apparences je passerai à une seule; d'un rêve très complexe à un rêve très simple. D'autres rêveront que je suis fou et moi je rêverai au

Zahir. Lorsque tous les hommes ici-bas penseront jour et nuit au Zahir, qui sera un songe et qui sera une réalité, la terre ou le

Zahir? » (Borges, p. 144) 
Roger-Michel Allemand - J'aimerais d'abord évoquer la place de l'humour dans votre œuvre. Comment intervient-il dans le processus de création?

Jean-Philippe Toussaint - Il y a eu quelques études sur le sujet $^{1}$, mais pour ce qui me concerne, l'humour est une des questions sur lesquelles je ne m'exprime pas volontiers. Autant j'ai des idées, des théories, sur beaucoup de points, autant je suis très conscient de ce que je fais, souvent - autant, sur l'humour, je préfère que cela reste instinctif, je ne veux pas trop le théoriser. La seule chose que je réponds, mais un peu pour éviter la question, c'est une sorte de boutade : l'humour, c'est comme l'espionnage, mieux vaut ne pas révéler ses méthodes pour ne pas nuire à l'efficacité de l'entreprise ${ }^{2}$. Je préfère donc essayer de rester efficace.

R.-M. A. — «En vérité, je m'étais mépris dès le début sur Jean-Christophe de G. D'abord, je n'ai cessé de l'appeler JeanChristophe alors qu'il s'appelle Jean-Baptiste [...] (se fût-il appelé Simon que je l'aurais appelé Pierre, je me connais). » (Toussaint, 2009, p. 75). À lire un tel passage, il y en a d'autres, on peut s'interroger sur la portée du clin d'œil évangélique. Référence amusante, (auto)dérision, signe de connivence en direction du lecteur, affirmation du narrateur en figure christique, ou autre chose?

J.-Ph. T. - L'ironie d'appeler Jean-Baptiste de Ganay «Jean-Christophe» me plaisait dans un sens en effet lié à

\footnotetext{
1 Voir les communications de Laurent Demoulin, «La fougère dans le frigo. L'humour chez Jean-Philippe Toussaint», et Patricia Frech, "L'humour dans l'œuvre de Jean-Philippe Toussaint », lors du colloque Les Écrivains minimalistes, Centre Culturel International de Cerisy-la-Salle, 21-31 juillet 2003.

2 Toussaint reprend ici l'aphorisme confié à Frech, 2002, p. 6.
} 
l'humour : une sorte de mauvaise foi, d'impertinence de la part du narrateur, qui se trouve en position de rivalité avec ce JeanBaptiste, ou ce Jean-Christophe. Il y a cette désinvolture affichée. Pour ce qui est du clin d'œil à l'évangile, c'est vrai que j'y ai réfléchi et à un moment je me suis demandé pourquoi Simon y était appelé «Pierre ». C'est quand même incroyable : on l'appelle ainsi et on apprend qu'en réalité, il se nomme autrement ! En fait, il n'y a pas d'explication, ou alors je n'ai pas très bien compris. J'avais même envisagé de mener des recherches pour en connaître la raison. Ce que je n'ai pas fait, car au fond, cela m'était égal. Mais la citation que vous donnez est un vestige de cette interrogation. Cela dit, indépendamment de la plaisanterie ou de l'impertinence, la question des noms propres m'intéresse beaucoup dans tous mes romans. Le personnage féminin du premier roman c'est Edmondsson, ce qui n'est pas un nom classique pour une jeune femme. De la même façon, je crois qu'il y a dans tous mes livres une recherche sur les noms propres. La Vérité sur Marie en est un nouvel exemple: appeler le personnage «Jean-Christophe de G. », c'est encore une réflexion du même ordre.

R.-M. A. - «Marie s'appelait de Montalte, Marie de Montalte, Marie Madeleine Marguerite de Montalte (elle aurait pu signer ses collections comme ça, M.M.M.M, en hommage sybillin à la maison du docteur Angus Killierankie). Marie, c'était son prénom, Marguerite, celui de sa grand-mère, de Montalte, le nom de son père (et Madeleine, je ne sais pas, elle ne l'avait pas volé, personne n'avait comme elle un tel talent lacrymal, ce don inné des larmes). Lorsque je l'ai connue, elle se faisait appeler Marie de Montalte, parfois seulement Montalte, sans la particule, ses amis et collaborateurs la surnommaient Mamo, que j'avais transformé en MoMa au moment de ses 
premières expositions d'art contemporain. Puis, j'avais laissé tomber MoMa, pour Marie, tout simplement Marie (tout ça pour ça). » (Toussaint, 2002, p. 54) : c'est drôle, bien sûr, mais pas seulement. Outre l'importance du rythme - la gradation ici, le décrochement ailleurs - et celle des parenthèses - comme toujours chez vous - , il y va, me semble-t-il, de l'inscription comme d'un cryptage, d'une distanciation.

J.-Ph. T. - C'est un prolongement de la réponse précédente: cela prouve l'importance que j'accorde aux noms propres. La citation entière est une démonstration. C'est truffé de références, en effet. Montalte est un des pseudonymes que Blaise Pascal utilisait dans Les Provinciales. Pascal, on le trouve dans tous mes romans. Déjà dans La Salle de bain, avec toute la réflexion sur le divertissement et aussi la citation de Pascal, en anglais. Il revient sous la forme du personnage féminin, Pascale, dans L'Appareil-photo. Même Marie maintenant est pascalienne, d'une certaine façon, par son nom de famille. Après, il y a un jeu sur les prénoms, mais je ne vais pas rentrer dans tous les détails. Cela montre en tout cas que je ne choisis pas les noms propres à la légère. Le passage que vous citez est tout à fait emblématique de ce point de vue. En même temps, c'est lourd de références, mais je voudrais que ce soit exprimé avec légèreté - comme j'y arrive, je crois, dans le paragraphe en question.

R.-M. A. - Pascal est une des influences que vous soulignez volontiers, avec celles de Musil, Beckett ou Borges. Quelles en sont les raisons?

J.-Ph. T. - La raison est assez fortuite au début. Pendant que j'étais en train d'écrire La Salle de bain, je me suis rendu compte que le personnage que je décrivais devait être assez proche des préoccupations de Pascal. Je n'avais pas lu cet 
auteur dans le détail et j'ai voulu le vérifier. J'étais en Algérie à l'époque et je me suis procuré un exemplaire des Pensées, que j'ai donc lu attentivement pour la première fois dans cette perspective. La référence pascalienne est très claire dans La Salle de bain ; ensuite de quoi, je l'ai cultivée.

R.-M. A. - Et le flacon d'acide chlorhydrique (Toussaint, 2002), à qui est-il adressé ?

J.-Ph. T. - Une réponse que je peux faire, c'est qu'il correspond à une intention littéraire consciente. Généralement, les éléments romanesques que j'utilise ne sont pas symboliques, au sens immédiat du terme. Ainsi, je ne pense pas que la salle de bain était un symbole. Je ne l'avais pas choisie comme telle. J'ai parlé de la salle de bain comme d'une pièce concrète, qui se trouvait avoir des références symboliques, mais ce n'était pas en tant que symbole que cela m'intéressait. Or l'acide m'intéressait en tant que tel. Après La Télévision, le livre le plus léger que j'avais écrit, je m'étais fait la réflexion que l'acidité de mes premiers livres avait disparu, et consciemment, j'ai eu envie de remettre un peu d'acide. Là, ce flacon, et c'est assez rare, a un sens symbolique.

R.-M. A. - De la littérature comme antidote au divertissement ${ }^{3}$ et à la télévision?

J.-Ph. T. - Ce n'est pas en ces termes que je me suis posé la question : il s'agissait pour moi d'opposer à la télévision, la vie. Et pas spécialement la littérature d'ailleurs. La vie. Et la littérature était un moyen à ce moment-là.

3 Voir Lepape, 1997. 
R.-M. A. - Dans Autoportrait (à l'étranger), vous soulignez la matière ironique de tous vos livres : à quelles fins, cette ironie?

J.-Ph. T. - Je vous ai fait part de ma réserve au début de notre entretien. Je n'ai pas de réponse.

R.-M. A. — Quel est donc l'objet de vos réticences ?

J.-Ph. T. - Disons qu'il s'agit davantage d'une réflexion sur le mot "réticence», qui a plusieurs sens. Le premier, habituel, c'est de ne pas avoir très envie, ne pas être très chaud, très partant, bref une sorte de mollesse dans le refus. Ce sens-là, il est évident, il me plaît - il me convient bien et va bien avec mes personnages -, mais il y en a aussi un autre qui m'intéresse : le manque, la chose qui n'est pas dite, l'omission. Le mot a donc beaucoup d'avantages d'un point de vue conceptuel. C'est en cela qu'il me plaisait de l'utiliser. J'aime bien qu'il soit ainsi, polysémique.

R.-M. A. - J'effleurai le jeu de vos parenthèses : il est difficile d'y distinguer (ce qui devrait vous convenir) entre l'incise anodine, la mention porteuse d'un sens plus ou moins caché, justement, et la fausse précision, en réalité porteuse d'incertitude (comme un décalage irréductible). Comment les concevez-vous?

J.-Ph. T. - Très brièvement, je pense que j'ai retenu une leçon littéraire de Nabokov, qui est d'employer les parenthèses avec une volonté ironique ou comique. Il les utilisait souvent pour placer une notation humoristique, en tout cas pour créer un "décalage », comme vous dites, un décrochement dans le texte. Très vite, j'ai fait de même. Dans mes derniers romans, en particulier les trois derniers, comme la tonalité est plus sombre 
et qu'il y a moins d'ironie, mais comme j'ai toujours besoin de ce décrochement, de cette cassure du rythme de la narration, j'ai beaucoup plus employé les tirets. Ceux-ci ont donc remplacé les parenthèses. Il en reste, mais un peu transformées, puisqu'elles sont moins systématiquement comiques qu'auparavant.

R.-M. A. - Nous parlions tout à l'heure d'onomastique. Or l'étalon de La Vérité sur Marie porte un nom qui a capté mon attention. De fait, le mot arabe zāhir signifie "explicite», «littéral», «évident», « manifeste »... En dehors de ses implications religieuses", ce qui m'intrigue, c'est le fait qu'il s'oppose en principe aux interprétations subjectives. Vous l'avez choisi pour cela?

J.-Ph. T. - Je l'ai trouvé chez Borges, dans la nouvelle Le Zahir. Et surtout, dans cette nouvelle, j'ai trouvé une phrase - en haut d'une page, à gauche, je ne sais plus - qui disait en substance : zahir veut dire "visible », mais surtout qu'une fois qu'on l'avait vu, on ne pouvait plus l'oublier. Il suffit d'une seule fois et c'est inoubliable. Quand j'ai lu cette phrase et comme j'avais déjà un cheval qui trottait quelque part dans mon travail, je me suis dit: "Voilà : le cheval s'appelle Zahir.» Cela s'est imposé à moi en lisant la nouvelle de Borges.

\footnotetext{
${ }^{4}$ Ce concept est aux fondements de l'une des cinq grandes écoles juridiques de l'islam; il est employé dans l'exégèse du Coran et de la Sunna, en particulier par le zahirisme sufi. Borges y fait allusion à la fin de sa nouvelle : « Pour se perdre en Dieu, les soufis répètent leur propre nom ou les quatrevingt-dix-neuf noms de Dieu jusqu'à ce que ceux-ci ne veuillent plus rien dire. Je souhaite ardemment parcourir cette route. Peut-être finirai-je par user le Zahir à force d'y penser et d'y repenser [...]. » (Borges, 1967, p. 144-145) Notons que le terme était également utilisé dans la gnose ésotérique de l'alchimie arabe, où zāhir, l'exotérique, le manifesté, est conçu en relation permanente avec son antonyme, bātin, l'ésotérique, le caché.
} 
R.-M. A. - Je ne peux m'empêcher de corréler sa disparition, "fondu, noir sur noir, dans les ténèbres », à "la quintessence du réel, sa moelle sensible [...], la vérité idéale». Et puis il y a cette nuit omniprésente dans votre œuvre. Fût-ce au prix d'un bond culturel, cela me fait songer au premier enseignement du Tao: "Obscurcir cette obscurité, voilà la porte de toute merveille. » (Lao-tseu, 1967, p. 11). Où en êtesvous de votre quête?

J.-Ph. T. - Si la nuit apparaît dans mes premiers livres, c'est très instinctivement. Il n'y a pas de raison objective, ou que je pourrais expliquer, ou de raison délibérée. Peut-être qu'à partir de Faire l'amour, c'est plus conscient... Dans ce livre, en tout cas, je voulais parler de la lumière. Je voulais que la lumière soit au cœur du livre et en particulier, évoquer la lumière du Japon, évoquer la lumière de Shikoku. Et donc très naturellement, pour en parler, il vaut mieux situer l'action de nuit, parce qu'il y a beaucoup plus de variations, ainsi que toutes les lumières artificielles, qu'on peut décrire. Il y a donc là quelque chose de délibéré, lié à la lumière : parce que je voulais l'évoquer, il fallait passer par la nuit. Mais c'est certain qu'il y a sans doute une obsession, irrationnelle ou instinctive, un plaisir de la nuit. La Réticence, par exemple, comporte aussi beaucoup de scènes de nuit, alors que je n'avais pas ce désir conscient d'évoquer la lumière. C'est une obsession un peu comparable à la présence de l'eau dans mes livres.

R.-M. A. - Puisque l'eau «favorise tout» (Lao-Tseu, 1967, p. 19), laissons donc aller l'esprit de géométrie: le théorème de Pythagore, les deux infinis de Pascal, l'acide dans la trousse de toilette, les multiples bains, de mer ou autres, l'imaginaire élémentaire, la recherche revendiquée d'une 
énergie, d'abord par l'humour puis purement romanesque : cela n'oriente-t-il pas vers une autre lecture de votre œuvre?

J.-Ph. T. - Il y a un certain nombre d'obsessions dans mes livres. Je peux les constater, mais je ne les théorise pas. L'eau en est une, capitale, extrêmement présente, mais il n'y a pas d'explication rationnelle à cela. C'est une obsession, ça me plaît, c'est mon désir, c'est mon plaisir. J'ai toujours éprouvé du plaisir à parler de l'eau, à la décrire, à faire des métaphores aquatiques, fluides, liquides, mouillées. C'est comme ça : j'en ai envie. Point.

R.-M. A. - Faire lire dans une salle de bain, n'était-ce pas déjà attribuer une vertu lustrale à la littérature ?

J.-Ph. T. - Pas d'autre commentaire.

R.-M. A. - Dans Fuir, la disparition inexpliquée de Marie est de l'ordre du précipité et la précipitation, tant des personnages que de l'action ou du temps, correspond à deux éléments saillants chez vous. D'une part, la tension entre l'immobilité et le mouvement effréné. Entre La Salle de bain et l'incessant « déplacement professionnel » dans Fuir, où réside la constante?

J.-Ph. T. - Je passe.

R.-M. A. - D'autre part, la précipitation est celle croissante de vos phrases, qui se sont considérablement allongées depuis vos débuts. Ce qui m'amène à relier le déplacement à son équivalent étymologique : quel rôle joue la métaphore dans les accélérations et décélérations de votre écriture?

J.-Ph. T. - Le rythme des phrases est très dépendant du rythme de l'action. Il est évident que pour décrire un homme 
étendu dans sa baignoire, on peut se contenter d'une phrase simple. Lorsqu'il faut décrire un cheval qui s'échappe dans un aéroport, il est certain que si la phrase fait deux pages, elle s'unit, elle se marie à l'action en cours. J'ai donc essayé de faire en sorte que ma phrase suive le rythme du cheval qui s'emballe et qui s'échappe. Ma phrase ne s'échappe pas, mais d'une certaine façon, elle s'emballe. C'est moi qui la chevauche, si j'ose la métaphore: je chevauche la phrase qui s'emballe et je continue de la contrôler, tout en étant vraiment secoué. Mais il faut être très bon cavalier, pour ne pas être simplement baladé, emporté. J'aime cette métaphore : bon cavalier, c'est-à-dire bon technicien, et je crois que techniquement je suis plus à même maintenant, mieux armé pour maîtriser de telles phrases, qui sont extrêmement complexes, même si elles s'écrivent souvent d'un seul élan, d'un seul jet. Car je les retravaille : dans une phrase qui fait deux pages ou plus, j'ajoute des éléments, dans un tumulte en mouvement. Je pense que cela nécessite une plus grande maîtrise technique, comme écrivain, que les phrases simples et courtes de La Salle de bain, qui finalement étaient rassurantes, pour moi qui voulait tout maîtriser. Avec ces phrases très courtes, je risquais moins de me casser la gueule. Lorsque je suis au sommet de cette phrase qui s'élance au galop en pleine nuit, je dois être assez costaud techniquement

R.-M. A. - Dans le belgicisme "s'encourir», il y a le réfléchi. Serait-ce vous qui vous encourez?

J.-Ph. T. - Je n'entre pas dans cette métaphore. Ou alors pas volontairement. Un mot sur l'utilisation de ce verbe, parce qu'il y a belgicismes et belgicismes. Il y en a qui ne sont pas beaux et qui sont même des fautes de français. Je les évite. Et puis il y a des belgicismes qui viennent du français plus ancien. 
C'est le cas de "s'encourir », qui est plus beau que "partir en courant ». Cela me plaisait d'utiliser ce verbe, certes comme une coquetterie, mais une coquetterie que j'assume parce qu'elle ajoute quelque chose à la langue. Ou plutôt c'est quelque chose que la langue française a perdu et que seuls les Belges avaient gardé, et que je restitue, que je remets dans le pot commun.

R.-M. A. - Et l'Héautontimorouménos ?

J.-Ph. T. - No comment.

R.-M. A. - À propos de vos phrases, si nous parlions de Proust?

J.-Ph. T. - C'est un compagnonnage constant. Ce n'est pas non plus une influence trop contraignante. Dans La Télévision, il en est fait mention plusieurs fois, ne serait-ce que par le personnage qui retraduit Proust en allemand. C'est un écrivain dont je me suis nourri. Quelque chose me plaît dans la précision... Non : plutôt dans la complexité de la description de certains sentiments, états d'âme, qui sont extrêmement complexes et qu'il arrive à exprimer de façon limpide, sans faire l'impasse d'aucune nuance, allant dans tous les détours. Il y a une sorte d'introspection psychologique de certaines phrases qui me plaît. Il y a aussi quelque chose qui est peut-être moins souligné, mais que j'aime beaucoup chez lui : son goût pour l'aphorisme. Ce n'est peut-être pas hypermoderne, en tout cas «vingt-et-unième siècle », mais ce n'est pas non plus La Rochefoucauld. Proust a une façon d'écrire les aphorismes, au milieu d'une phrase, qui est encore utilisable aujourd'hui. Alors qu'écrire des aphorismes dans un recueil, je ne sens pas trop la façon de le faire. Les aphorismes de Proust sont 
englobés dans la prose et passent très bien. C'est une leçon que j'ai retenue.

R.-M. A. - Revenons à l'idée de décrochement stylistique, qui va de pair avec le changement subit de registre - « Le jour se levait sur Tokyo, et je lui enfonçais un doigt dans le trou du cul. » (Toussaint, 2002, p. 91). À quoi correspond ce trait ?

J.-Ph. T. - En fait, il faudrait faire partir la citation de beaucoup plus loin. Ce qui est saisissant dans la fin de la phrase, c'est le contraste avec presque toute la première partie du livre. $\mathrm{Si}$ on la cite hors contexte, elle est dénaturée. C'est une phrase très forte parce que la fin est une sorte d'éclair qui illumine en contrepoint tout ce qui précède, au moins les deux pages qui précèdent. J'ai beaucoup travaillé ce passage. Notamment, je n'arrivais pas à ce «doigt dans le trou du cul», si je puis dire. J'avais un énorme problème, j'y revenais sans cesse et cela ne marchait jamais, parce que le «doigt dans le trou du cul» arrivait toujours à la fin d'une très longue phrase, qui faisait deux pages. J'ai trouvé la solution en me disant qu'il fallait couper la phrase. Si c'était trop amené par les deux pages avant, cela ne marchait jamais. Il fallait donc l'amener, puis mettre un point et : « Le jour se levait» - comme un changement de plan au cinéma - « et je lui enfonçais un doigt dans le trou du cul. » L'image est alors saisie en une fois. Alors que si c'est amené par les deux pages qui précèdent, on n'arrive pas à saisir l'image en une fois. Il y a donc toute la préparation, qui est là comme un système de persistance cérébrale, et puis l'image, qui est seule. J'y ai travaillé deux mois, alors je l'explique volontiers. Au terme de ces deux mois, j'ai optimisé la façon de faire sonner la phrase. 
R.-M. A. - Le contraste est travaillé entre des représentations triviales et une tension vers le sublime, rejeté en même temps qu'avancé: "Je regardais l'immense étendue de la ville derrière la baie vitrée, et j'avais le sentiment que c'était la terre elle-même que j'avais sous les yeux, dans sa courbe convexe et sa nudité intemporelle, [...] et j'eus alors fugitivement conscience de ma présence à la surface de la terre, impression fugace et intuitive qui, dans le douceâtre vertige métaphysique où je vacillais, me fit me représenter concrètement que je me trouvais à l'instant quelque part dans l'univers. » (Toussaint, 2002, p. 47), que l'on retrouve dès la première phrase de La Mélancolie de Zidane : «Zidane regardait le ciel de Berlin sans penser à rien, un ciel blanc nuancé de nuages gris aux reflets bleutés, un de ces ciels de vent immenses et changeants de la peinture flamande, Zidane regardait le ciel de Berlin au-dessus du stade olympique le soir du 9 juillet 2006, et il éprouvait avec une intensité poignante le sentiment d'être là, simplement là, dans le stade olympique de Berlin, à ce moment précis du temps, le soir de la finale de la Coupe du monde de football. ». Chez Toussaint, qu'y at-il donc après la physique?

J.-Ph. T. - Je passe. C'est trop complexe.

R.-M. A. - Un regard tourné vers la terre, un autre vers le ciel. Aristote et Platon : L'École d'Athènes dans la Chambre des Signatures?

J.-Ph. T. - Je n'avais pas pensé à cette métaphore. Il faut dire que L'École d'Athènes est très référencée et lourdement symbolique. Je ne pense pas que je le sois jamais, mais je ne vais pas critiquer L'École d'Athènes.

R.-M. A. - Dans Fuir, le champ lexical de la permanence était récurrent. Dans Faire l'amour, vous évoquez l'impermanent (Toussaint, 2002, p. 155). Où en êtes-vous à ce sujet? 
J.-Ph. T. - Permanence, impermanence... Non, je n'ai pas grand-chose à en dire.

R.-M. A. - Faire l'amour est cependant le premier de vos livres où vous avez "osé» la psychologie et l'adjectivité, à l'encontre de vos débuts, d'influence néo-romanesque. Au début des années 1990, Alain Robbe-Grillet me disait même qu'il vous tenait pour son principal héritier, au point de donner des cours sur L'Appareil-photo. Que s'est-il passé qui a infléchi votre écriture?

J.-Ph. T. - Oui, je sais que Robbe-Grillet faisait un cours sur L'Appareil-photo, en particulier sur le premier paragraphe, qui est très programmatique. C'est vrai qu'il $\mathrm{y}$ a matière. Ce n'est pas du tout un essai, mais c'est tellement conceptuel et proche de la théorie que cela se prête beaucoup à l'étude. À partir de Faire l'amour, il n'y a dans mon esprit aucun renoncement à quoi que ce soit, je ne renonce à rien, mais je ne me prive plus de rien non plus. Dans mes premiers livres, je me privais d'un certain nombre d'éléments, en particulier de la psychologie, parce que je m'en méfiais et parce qu'il me semblait qu'utilisés abusivement, ils étaient lourds et inintéressants, et nous éloignaient de ce qui était le sens véritable de la littérature, qui est une question de forme et de rythme. Je me méfiais de ce type de béquille et refusais donc la psychologie, sans doute dans la lignée de Pour un nouveau roman. À partir de Faire l'amour, je me suis dit que je n'avais à me priver de rien, que je pouvais utiliser tout ce qui est à la disposition d'un écrivain. Et justement, comme j'use de la psychologie avec parcimonie, elle ajoute alors quelque chose. Cela n'enlève rien et je reste fidèle à moi-même. Pour moi, c'est très important de dire cela. Il n'y a aucun renoncement. C'est simplement poursuivre une recherche et, en poursuivant cette 
recherche, ne plus avoir peur de certains éléments qui seraient prétendument interdits ou à éviter. Votre mot d'infléchissement est juste. Nous sommes d'accord. Il faut pouvoir tout utiliser.

R.-M. A. - À propos de Robbe-Grillet, vous savez que lorsqu'un récit lui venait, il savait aussitôt s'il entreprendrait un livre ou un film, selon ce qui se présentait à son esprit: structures de langage ou images et son ${ }^{5}$. Tel n'est apparemment pas votre cas, puisque, pour le dire vite, vos derniers romans sont visuels. Je pense en particulier aux jeux de lumière dans Faire l'amour, à l'importance des couleurs dans ce roman et dans le suivant, à la course poursuite à travers Pékin dans Fuir, ou à la scène sur le tarmac de Narita dans La Vérité sur Marie (Toussaint, 2009, p.84-138). Pour autant, ce n'est pas du cinéma: la puissance des images s'impose d'emblée sous une forme très littéraire et difficilement transposable à l'écran. Comment envisagez-vous la comparaison entre vos activités de réalisateur et d'écrivain?

J.-Ph. T. - Votre question dit très bien les choses, alors je ne vais pas les répéter. C'est vrai que mon imagination est toujours visuelle, essentiellement visuelle. Hier, je me suis même prêté à une expérience où, par résonance magnétique, on prenait des images de mon cerveau. On m'a posé un certain nombre de questions: "Avez-vous ressenti des odeurs? Non. - Avez-vous entendu des sons ? - Non. - Avez-vous vu des images? - Oui. » Sur une échelle de un à dix, j'avais toujours dix en visuel. Étalé dans cette espèce de scanner, je voyais des images. Mon cerveau fonctionne ainsi, ce qui me permet d'écrire des livres, de prendre des photos, de réaliser des films. La musique, ce n'est pas très visuel - encore que, on

5 Voir Robbe-Grillet, 2000. 
devrait pouvoir y arriver. Pour moi, l'imagination est visuelle, mais les moyens sont très différents, et je ne vais pas rentrer dans les détails de ce qui différencie la littérature du cinéma.

R.-M. A. - Plutôt que de cinéma, qui implique ipso facto le mouvement, ne vaudrait-il pas mieux souligner que vos images sont arrêtées, non seulement comme les instantanés de L'Appareil-photo, mais surtout telles des compositions picturales. Les références à Mondrian dans La Salle de bain, au Titien dans La Télévision, à Mapplethorpe dans Faire l'amour, et le métier de MoMa le signalent obligeamment. Quels rapports entre votre écriture et la peinture?

J.-Ph. T. - C'est une très vaste question. Je n'ai pas vraiment de réponse. Il faudrait peut-être que j'écrive un jour un texte là-dessus, que je m'y penche à l'occasion d'un entretien uniquement consacré à la question. Je n'ai pas envie de le faire maintenant en deux mots. C'est vrai que la peinture joue un rôle très important dans mes livres. On pourrait même aller au-delà des exemples que vous en donnez. Dans les derniers livres en particulier, on pourrait presque dire que ce sont des tableaux. Lorsque Zahir descend du van sur l'aéroport, on pourrait imaginer une très grande toile, à la Delacroix ou à la Géricault. Un très grand format, de nuit, quelque chose de dramatique. Il y a quelque chose à creuser, mais je n'ai pas envie de développer, encore que je vienne d'amorcer une réflexion. Il faudra que je me penche davantage sur la question. D'autant que vous n'êtes pas le seul à me la poser. Il y a un ou deux mois, une chercheuse m'en a parlé. Il y a aussi un bibliothécaire à Vienne qui m'a livré une intuition intéressante. Il voulait voir les rapports de mes livres avec le dessin et avec la peinture, et m'avait dit: "Peut-être qu'on pourrait faire cette hypothèse que vos premiers livres, 
jusqu'à La Télévision, se rapprochent du dessin et de l'esquisse, et que les derniers se rapprochent des grands tableaux. »

R.-M. A. - Pour en finir avec Alain, j'ai été un peu étonné que certains chroniqueurs aient décelé son ombre, ou cru le faire, dans La Vérité sur Marié 6 . Il me semble pourtant qu'il y a une différence de taille entre vos deux univers, non pas seulement le traitement des personnages, mais le fait d'une présence esthétique absolue : il n'y avait pas chez lui la beauté qu'il y a chez vous. Et je ne parle pas que du style et de la langue. Cela ne tient-il pas à la sensualité de votre inspiration?

J.-Ph. T. - Vous avez raison: c'est un malentendu complet de dire que La Vérité sur Marie est robbe-grillétien ce qui n'est en rien une critique de Robbe-Grillet. Certes, on a beaucoup souligné la description des chaussures ${ }^{7}$, mais elle peut faire aussi penser à la description de la casquette de Charles Bovary8. C'est déjà chez Flaubert, cette façon de s'attacher à certains détails. La question théorique, qui était présente dans Fuir déjà, où je me sens très proche et continue de me sentir très proche de Robbe-Grillet, c'est sa façon d'insister sur l'importance du manque. Je ne sais pas si c'est aussi clairement dit dans Pour un nouveau roman, mais il y a un chapitre sur l'importance du manque dans Préface à une vie d'écrivain, qui me semble un chapitre très important d'un point de vue théorique et m'a conforté, confirmé dans des intuitions que j'avais déjà: le manque est extrêmement dynamique, porteur, dans la littérature. Plus il y a de manque, plus il y a dynamisme et potentialités. Robbe-Grillet l'exprime très bien

\footnotetext{
6 Voir notamment Kapriélian, 2009.

7 Voir Toussaint, 2009, p. 45-46.

8 Voir Flaubert, 2001, p. 47.
} 
dans Préface à une vie d'écrivain et, pour moi, cela reste une leçon vivante et que j'utilise. Mais ce serait un malentendu de dire que je décrirais de façon obsessionnelle et maniaque.

R.-M. A. - Dès l'Antiquité, libido désigne aussi bien les appétits que la fantaisie ou l'excès. Chez Jung, le mot renvoie à l'énergie vitale. Le titre Faire l'amour et le verbe vivre, dans Fuir, ne résonnent-ils pas comme une double injonction?

J.-Ph. T. - Pas vraiment. Ni Fuir, ni Faire l'amour ne sont des injonctions. Sinon j'aurais dit: Faites l'amour! et Fuyez! Avec le point d'exclamation.

R.-M. A. - Vous définissez volontiers l'inspiration en termes de temps, de lumière, d'amour et de mélancolie: comment se conjoignent-ils dans la forme?

J.-Ph. T. - Je ne suis pas sûr que ce soit exactement tel qu'exprimé dans votre question. Mais ces mots me sont chers, tous. J'aime bien mettre ensemble forme et mélancolie, par exemple. "Il ne fut question ce soir-là que de forme et de mélancolie », ai-je écrit dans La Mélancolie de Zidane. Après avoir réfléchi à cette phrase, je me suis dit que c'était vrai pour Zidane, que c'était vrai pour La Mélancolie de Zidane aussi, puis j'ai élargi à tous mes livres et à toute ma recherche. Finalement, il n'y fut question que de forme et de mélancolie. Au-delà de ce livre-là, je trouve que cela peut être un résumé de ma recherche.

R.-M. A. - Me rappelant « encore qu'aux mots, il préférait la lumière » (Toussaint, 1986, p. 102), je me suis dit que nous pourrions terminer par une question démesurée: pourquoi écrivez-vous donc? 
J.-Ph. T. - La conclusion venait avec la mélancolie de ma précédente réponse.

R.-M. A. - C'est bien ce que j'avais compris. À quand l'automne-hiver?

J.-Ph. T. - Ça vient. J'y viens.

Liège, le 11 janvier 2011

\section{Bibliographie}

BORGES, Jorge Luis. 1967, L'Aleph, traduit de l'espagnol par Roger Caillois et René L. F. Durand, Paris, Gallimard, coll. «L'Imaginaire » : Le Zahir, p. 131-145.

FlaUbert, Gustave. 2001, Madame Bovary, Paris, Gallimard, coll. «Folio ».

FRECH, Patricia. 2002, L'Humour chez Jean-Philippe Toussaint, mémoire de maîtrise, Dijon/Mainz, Université de Bourgogne/Johannes Gutenberg Universität, août.

KAPRIÉLIAN, Nelly. 2009, " "Le plus fort dans un roman, c'est ce qui manque" », Les Inrockuptibles, $\mathrm{n}^{\circ} 721,22$ septembre.

LAO-TSEU. 1967, Tao-tö king, traduit du chinois par Liou Kiahway, Paris, Gallimard, coll. « Folio ».

LEPAPE, Pierre. 1997, « Pascal qui rit », Le Monde, 17 janvier.

RobBe-Grillet, Alain. 1963, Pour un nouveau roman, Paris, Minuit, coll. « Critique »; 
—. 2000, «L'écrivain à l'écran», conférence (Oxford, 25 septembre 1996) transcrite et mise en forme par RogerMichel Allemand, dans In the Temple of Dreams: The Writer on the Screen (Édouard d'Araille éd.), London, Living Time Press;

-. 2005, Préface à une vie d'écrivain, Paris, Seuil/France Culture, coll. «Fiction \& Cie ».

ToussainT, Jean-Philippe. 1985, La Salle de bain, Paris, Minuit;

—. 1986, Monsieur, Paris, Minuit;

-. 1989, L'Appareil-photo, Paris, Minuit;

—. 1991, La Réticence, Paris, Minuit;

—. 1997, La Télévision, Paris, Minuit;

—. 2000, Autoportrait (à l'étranger), Paris, Minuit;

-. 2002, Faire l'amour, Paris, Minuit;

-. 2005, Fuir, Paris, Minuit;

-. 2006, La Mélancolie de Zidane, Paris, Minuit;

—. 2009, La Vérité sur Marie, Paris, Minuit. 